\title{
Preclinical Pharmacokinetic Considerations for the Development of Antibody Drug Conjugates
}

\author{
Amrita V. Kamath • Suhasini lyer
}

Received: 29 July 2014 / Accepted: 20 November 2014 / Published online: 2 December 2014

(C) The Author(s) 2014. This article is published with open access at Springerlink.com

\begin{abstract}
Antibody drug conjugates (ADCs) are an emerging new class of targeted therapeutics for cancer that use antibodies to deliver cytotoxic drugs to cancer cells. There are two FDA approved ADCs on the market and over 30 ADCs in the clinical pipeline against a number of different cancer types. The structure of an ADC is very complex with multiple components and considerable efforts are ongoing to determine the attributes necessary for clinical success. Understanding the pharmacokinetics of an ADC and how it impacts efficacy and toxicity is a critical part of optimizing ADC design and delivery i.e., dose and schedule. This review discusses the pharmacokinetic considerations for an ADC and tools and strategies that can be used to evaluate molecules at the preclinical stage.
\end{abstract}

KEY WORDS antibody drug conjugate - biotherapeutics . cancer · pharmacokinetics · preclinical

\section{INTRODUCTION}

Antibody drug conjugates (ADCs) are a promising class of antibody related therapeutics for cancer that combine the antigen targeting specificity and favorable pharmacokinetic properties of monoclonal antibodies with the cytotoxic potential of small molecule chemotherapeutics (1-3). The vision of ADCs is to provide targeted delivery of the cytotoxic agent to tumor tissue and spare normal tissue, thereby decreasing its toxicity and improving its therapeutic window. The design of an $\mathrm{ADC}$ is critical in delivering on this vision and there is a lot of research focused on the optimal design of the molecule and its main components i.e., the antibody directed to an antigenic target, the cytotoxic drug and the linker that attaches the

A. V. Kamath $(\square) \cdot$ S. lyer

Department of Preclinical and Translational Pharmacokinetics and

Pharmacodynamics, Genentech, Inc, I DNA Way (Mailstop 463A)

South San Francisco, CA 94080, USA

e-mail: kamath.amrita@gene.com antibody to the drug (4-6). Some considerations for each component (antibody, linker, drug) as well as the molecule as a whole are highlighted in Fig. 1. An important question in the development of ADCs is to define the exposure-response relationship for both efficacy and safety. Understanding the pharmacokinetics of the ADC, exposure at the site of action and drivers of efficacy and toxicity are important to address this key question, to further enable the design of a better molecule. Additionally, this can be used for optimizing dose and regimen to help realize the promise of an ADC therapeutic.

Mylotarg® (gemtuzumab ozogamicin) was the first ADC to be approved in 2000 for the treatment of acute myeloid leukemia (AML) and was composed of a CD33-targeted antibody linked to the cytotoxic drug calicheamicin via an acidlabile hydrazone linker. It was later withdrawn from the market in 2010 over concerns of safety and failure to reproduce its clinical benefit. There are currently two FDA approved ADGs on the market, Adcetris ${ }^{\circledR}$ (brentuximab vedotin) approved in 2011 for the treatment of Hodgkin's Lymphoma and anaplastic large-cell lymphoma, and Kadcyla ${ }^{\circledR}$ (ado-trastuzumab emtansine) approved in 2013 for the treatment of HER+ metastatic breast cancer. Adcetris ${ }^{\circledR}$ is a CD30-targeted antibody linked to an auristatin (monomethyl auristatin E, MMAE) via a protease cleavable linker, and Kadcyla ${ }^{\circledR}$ is a HER2-targeted antibody (trastuzumab) linked to a maytansinoid derivative (DM1) via a non-cleavable thioether linker.

The clinical pipeline has more than $30 \mathrm{ADCs}$ at various stages of development from Phase 1 to Phase 3 and many more ADCs at the preclinical stage $(7,8)$. The field is rapidly evolving and tremendous effort is being put into applying insights from more advanced ADGs to guide the design of next generation ADCs. Some of the modifications being explored include novel cytotoxins, linkers, different sites of conjugation, and antibodies to novel antigenic targets. Several design features of an ADC impact its pharmacokinetics that 
Fig. I Desired attributes of the components of an ADC.

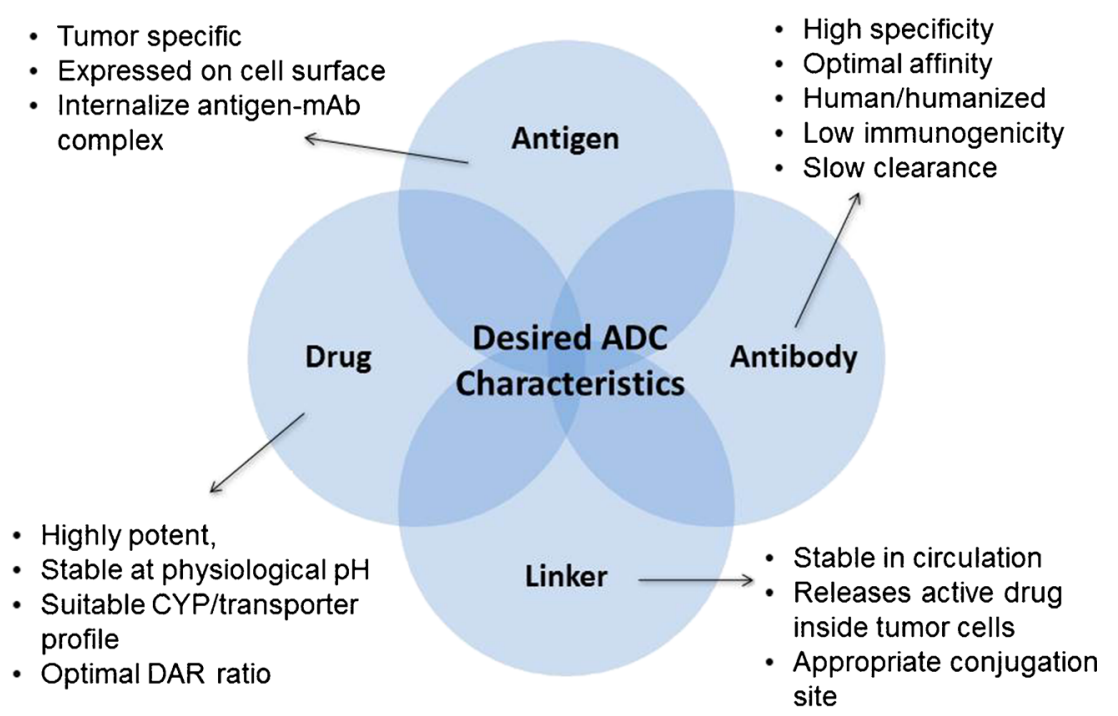

could then impact its efficacy and toxicity $(5,9)$. One important example is the choice of linker which ideally should be stable in circulation, but release the active drug inside the tumor cell. The types of linkers being explored are cleavable or non-cleavable, with varying degrees of stability. The site of conjugation on the antibody also has an impact on stability of the linker with different sites conferring varying degrees of stability to the ADC.

In this review we discuss the pharmacokinetic considerations in the development of ADCs and the strategies and tools that can be employed to evaluate them at the preclinical stage. We also briefly discuss the bioanalytical considerations and commonly used methods for pharmacokinetic assays.

\section{BIOANALYTICAL CONSIDERATIONS}

In addition to being complex molecules, ADCs are also heterogeneous mixtures comprising of multiple species with varying numbers of drugs per antibody (drug to antibody ratio, DAR) as well as different sites of drug linkage arising from different conjugation chemistry approaches such as conjugation through lysines (Kadcyla $\left.{ }^{\circledR}\right)$ or cysteines derived from reduced internal disulfide bonds (Adcetris $\left.{ }^{\circledR}\right)$, or site specific conjugation (10). These heterogeneous and dynamic characteristics of an ADC result in a unique set of bioanalytical challenges requiring multiple bioanalytical assays. In order to adequately characterize the pharmacokinetics of an $\mathrm{ADC}$, and answer the key question on exposure-response relationships, it is critical to understand what analytes are relevant, what needs to be measured, and at what stage of development. The bionalytical strategies for the development of ADCs have been the subject of intense discussion and are highlighted in several recent papers including a comprehensive review by Kaur and colleagues at Genentech and a white paper by the
ADC working group of the American Association of Pharmaceutical Scientists (11-13).

The analytes commonly used for evaluation of ADC PK and their associated PK profiles are shown in Fig. 2 (9,11). They include antibody related analytes such as i) total antibody (Tab) which measures both conjugated and unconjugated antibody and ii) conjugated antibody which measures antibody that has at least one drug attached to it (i.e., $\geq$ DAR 1), and small molecule related analytes such as i) antibody conjugated drug which measures any drug associated with the antibody and ii) unconjugated drug. The analytical methods used to measure these analytes include ELISA as well as LC/MS/MS methods.

Additional complexity is introduced by in vivo biotransformation of an ADC due to deconjugation and catabolism. This results in changing of the initial DAR distribution caused by loss of one or more drugs, as well as formation of several new species such as catabolites and metabolites, linkers and linker drugs, adducts with endogenous molecules such as albumin, cysteine, and complexes with any soluble/shed target antigen and other antibodies (11). Since the DAR distribution can continue to change in vivo, with possible impact to the clearance, distribution, and activity of the $\mathrm{ADC}$, it is important to develop appropriate methods to measure this. Novel bioanalytical assays have been developed to characterize DAR distribution such as affinity capture capillary LC-MS and affinity capture hydrophobic interaction chromatography (HIC) (11,14-16). In addition to DAR distribution, these assays can also provide insights into the mechanisms of drug loss e.g., maleimide exchange and formation of adducts with albumin or cysteine for ADCs using maleimide chemistry $(11,17)$.

At the preclinical stage, when the ADC design is still being optimized, it is valuable to use all these assays to characterize the pharmacokinetics as well as gain a mechanistic 
Fig. 2 Pharmacokinetic profiles of different analytes.

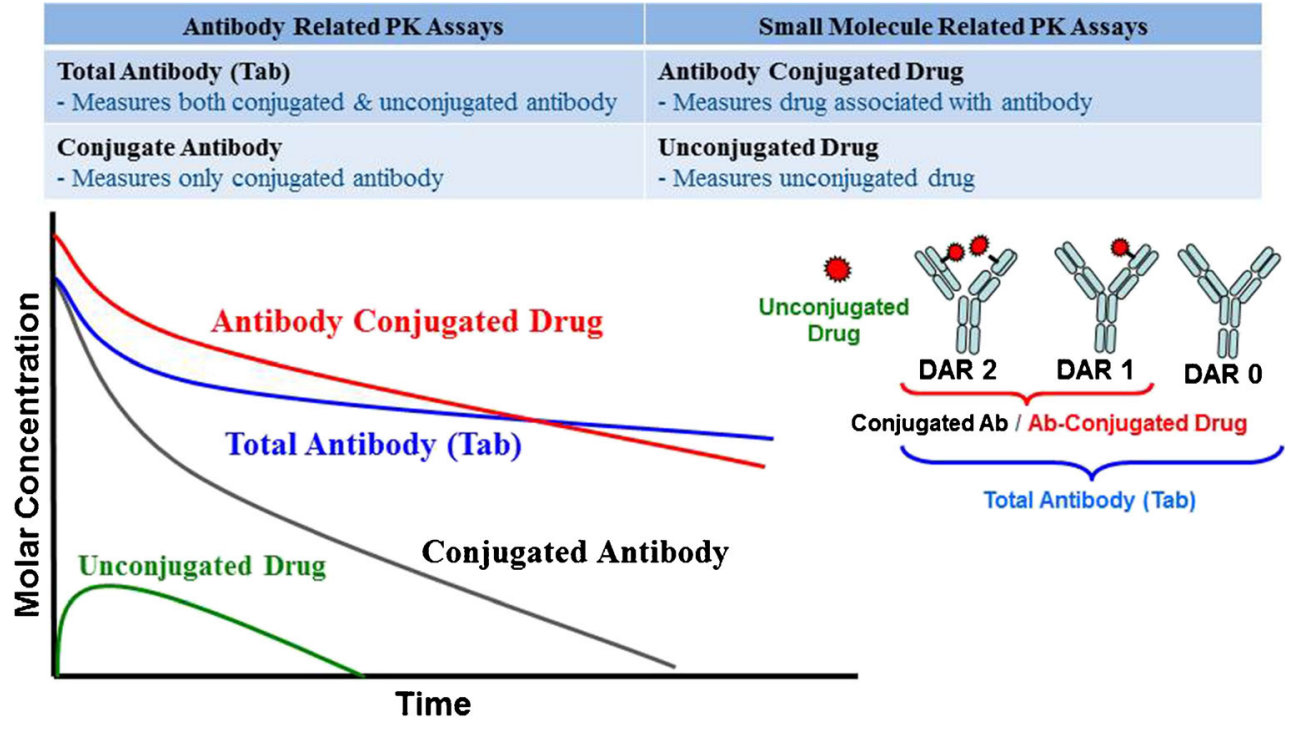

understanding of deconjugation and catabolism processes. With further development and availability of more information on the mechanisms of biotransformation, exposureresponse relationships, as well as the correlation between various analytes, the types of bioanalytical assays employed could be determined on a case-by case basis depending on the type of information required at that stage (13).

\section{PHARMACOKINETIC PROPERTIES OF ADCS}

The primary mechanism of action of an ADC is binding to its specific antigen target on tumor cells and internalization via receptor mediated endocytosis followed by trafficking from endosomes to the lysosomes where the cytoxic drug is released into the cell causing cell death (1). The ADC however can also be taken up into cells (with or without target expression) nonspecifically via pinocytosis, which could lead to unwanted drug release in non-target cells. Such non-specific uptake and release of potent drug could contribute to toxicity. The theoretical ADC elimination pathways based on nonclinical and clinical data are shown in Fig. 3 and include deconjugation and degradation or catabolism through nonspecific or target-mediated proteolysis (9). These processes could take place to varying extents in circulation and/or intracellularly depending on the characteristics of the ADC components $(5,17,18)$. Deconjugation of the ADC leads to the formation of unconjugated antibody and unconjugated drug, while catabolism of the ADC leads to the formation of antibody fragments or drug containing catabolites. The unconjugated antibody or antibody fragments can further undergo proteolysis to generate/release amino acids. The cytotoxic drug and drug related catabolites can undergo metabolism via CYP or non-CYP enzymes or be transported by transporters like P-gycoprotein and get excreted via the biliary or renal route.

The overall PK characteristics of an ADC such as slow clearance, long half-life and limited tissue distribution are driven by its antibody component. In addition to the antibody component, there are additional elimination mechanisms related to its linker and drug component. The characteristics of an ADC that can influence its pharmacokinetics and factors that should be considered during its preclinical development are shown in Table I and are discussed in more detail below. The PK parameters with the conjugated antibody analyte of Adcetris ${ }^{\circledR}$ and Kadcyla ${ }^{\circledR}$ across multiple species are shown in Table II.

\section{Antibody}

Characteristics related to antibody biology that affect the PK of an ADC include antibody structure, binding affinity and specificity to the antigen, FcRn binding, and Fcgamma interaction that drive effector functions $(23,24)$. The relatively long life of an ADC compared to a small molecule is due to recycling via $\mathrm{FcRn}$, which protects it from catabolism in the lysosomes. As with antibodies, the ADC can be taken up into cells specifically via receptor mediated endocytosis (targetdependent mechanism) or non-specifically via pinocyctosis (target-independent mechanism). FcRn-bound ADC can be recycled back to the cell surface and released back into circulation while ADC that is not bound to FcRn undergoes proteolytic degradation in the lysosome. Another similarity of ADCs to antibodies is the phenomenon of target mediated drug disposition where the interaction of the antibody with the target antigen impacts its pharmacokinetics including its clearance and distribution (25). This typically results in non-linear PK with higher clearance at lower doses and a decrease in the clearance at higher doses once the target is saturated. Also 
Fig. 3 Proposed disposition of an ADC.

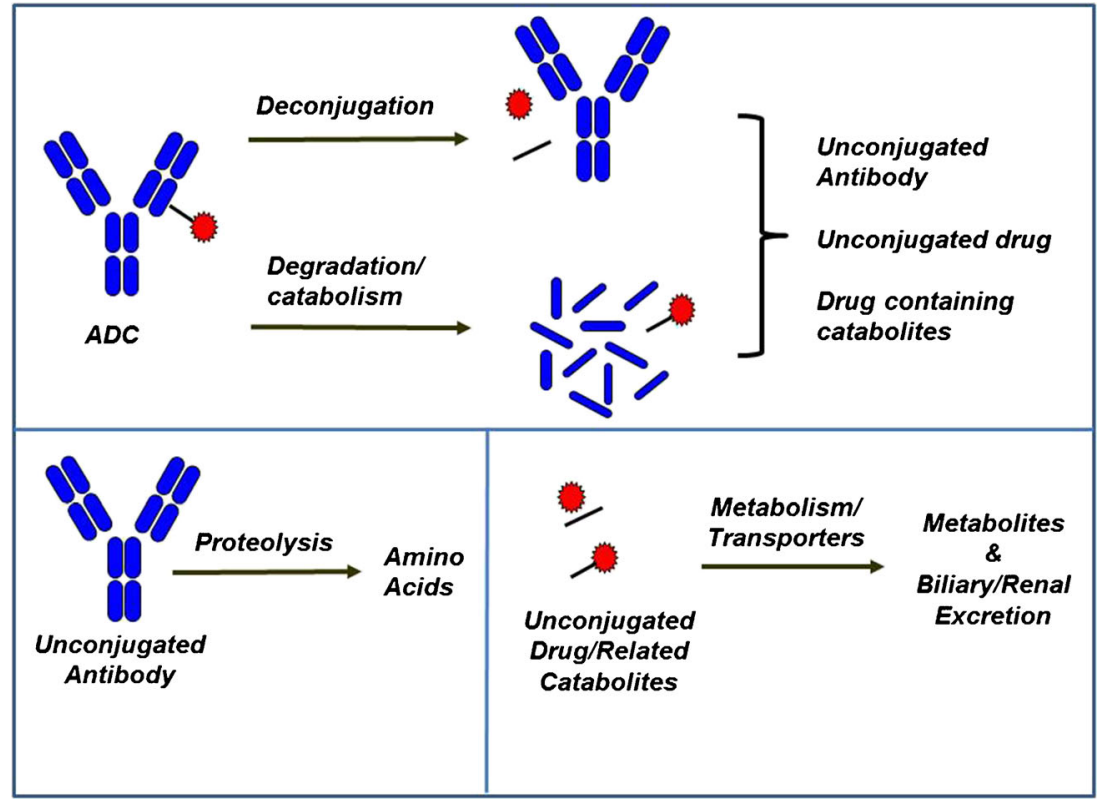

similar to an antibody, the tissue distribution of an ADC is limited with the initial distribution in the vascular space followed by slow diffusion across vascular endothelial cells into tissues. This can also be influenced by binding to and internalization by the target antigen $(23,24)$. Other factors that impact $\mathrm{PK}$ and tissue distribution of an ADC that are similar to an antibody are i) presence of soluble and/or shed antigen in circulation that can form immune complexes on binding to the ADC (26,27), and ii) immunogenicity to the ADC (formation of anti-therapeutic antibodies, ATAs) that can increase its clearance and decrease exposure (24).

The selection of the right antibody that has pharmacokinetic properties that are consistent with expected
IgG behavior is very crucial to ensure optimal PK properties of the ADC. The pharmacokinetics of an antibody may be altered by the conjugation of the drug (28) and this impact can be discerned by comparing the PK profiles of the naked antibody and the Tab of the $\mathrm{ADC}$ as shown in Fig. 4. The further apart the two curves, the larger is the impact of conjugation on the antibody. Conjugation can also impact the tissue distribution as has been observed for some ADGs when compared to the naked antibody $(29,30)$. Several factors that could contribute to this change including drug load, hydrophobicity among others have been investigated and are discussed in more detail below.

Table I Influence of ADC Characteristics on its Pharmacokinetics

\begin{tabular}{|c|c|c|}
\hline $\begin{array}{l}\text { ADC } \\
\text { characteristic }\end{array}$ & PK considerations & Assessment \\
\hline Antibody & $\begin{array}{l}\text { PK of naked antibody should be consistent with expected } \\
\text { lgG PK (non-target mediated disposition) }\end{array}$ & $\begin{array}{l}\text { Characterize PK-dose dependency: target affinity, target } \\
\text { expression/turnover, no off-target binding }\end{array}$ \\
\hline Linker & $\begin{array}{l}\text { i) Conjugation can impact the PK of an antibody depending on } \\
\text { the type of conjugation chemistry utilized. } \\
\text { ii) Linker should have suitable stability to deliver ADC to target to } \\
\text { minimize toxicity but have sufficient lability to release active drug once } \\
\text { internalized }\end{array}$ & $\begin{array}{l}\text { i) Assess impact of conjugation of the PK of the naked antibody } \\
\text { ii) Assess linker stability in vitro and in vivo } \\
\text { iii) Evaluate what is being released (active drug, catabolites) } \\
\text { iv) Evaluate mechanism for instability across species }\end{array}$ \\
\hline $\begin{array}{l}\text { Site of } \\
\text { conjugation }\end{array}$ & $\begin{array}{l}\text { Conjugation at some sites stabilizes the linker possibly due } \\
\text { to differences in solvent accessibility and local charge. }\end{array}$ & Assess linker stability in vitro and in vivo. \\
\hline $\begin{array}{l}\text { Drug load } \\
\text { (DAR) }\end{array}$ & $\begin{array}{l}\text { Higher DAR species associated with faster clearance and increased } \\
\text { toxicity }\end{array}$ & Assess in vivo pharmacokinetics and tissue distribution \\
\hline Cytotoxic drug & $\begin{array}{l}\text { i) MOA of the drug can impact the PK driver of efficacy/toxicity } \\
\text { e.g. tubulin binding agents vs. DNA damaging agents } \\
\text { ii) Release of the active drug and any relevant metabolites could } \\
\text { also impact PK drivers }\end{array}$ & $\begin{array}{l}\text { i) Assess metabolites, DDI potential (CYP inhibition/ induction/ } \\
\text { reaction phenotyping) } \\
\text { ii) P-gp substrate/inhibitor (other transporters) }\end{array}$ \\
\hline
\end{tabular}


Table II ADC Pharmacokinetic parameters of Kadcyla $®$ and Adcetris ${ }^{\circledR}$ in Rat, Monkey, and Human

\begin{tabular}{|c|c|c|}
\hline Characteristic & Kadcyla ${ }^{\circledR}$ & Adcetris ${ }^{\circledR}$ \\
\hline Target & HER2 & CD30 \\
\hline Antibody isotype & $\lg G \mid$ & $|g G|$ \\
\hline Cytotoxic drug & $\mathrm{DMI}$ & MMAE \\
\hline Linker & $\begin{array}{c}\text { MCC (thioether- } \\
\text { noncleavable) }\end{array}$ & $\begin{array}{l}\text { MC-vc-PAB } \\
\quad \text { (protease cleavable) }\end{array}$ \\
\hline Avg DAR & 3.5 & 4 \\
\hline \multicolumn{3}{|c|}{ Pharmacokinetic parameters (Analyte: conjugated antibody) } \\
\hline \multicolumn{3}{|c|}{ Rat } \\
\hline $\begin{array}{l}\text { Dose } \\
\text { CL (mL/day/kg) } \\
\text { TI/2 (day) } \\
\text { Vss }(\mathrm{mL} / \mathrm{kg})\end{array}$ & $\begin{array}{l}20 \mathrm{mg} / \mathrm{kg} \text { single IV dose } \\
13-15 \\
4.6 \\
62-64\end{array}$ & $\begin{array}{l}5 \mathrm{mg} / \mathrm{kg} \text { single } \mathrm{N} \text { dose } \\
19.8 \\
8-15 \\
-\end{array}$ \\
\hline \multicolumn{3}{|l|}{ Monkey } \\
\hline $\begin{array}{l}\text { Dose } \\
\mathrm{CL}(\mathrm{mL} / \text { day/kg) } \\
\mathrm{TI} / 2(\text { day }) \\
\text { Vss }(\mathrm{mL} / \mathrm{kg})\end{array}$ & $\begin{array}{l}30 \mathrm{mg} / \mathrm{kg} \mathrm{IV} \mathrm{q} 3 \mathrm{~W} \\
9.4-10.5 \\
4.6-5.2 \\
68-70\end{array}$ & $\begin{array}{l}3 \mathrm{mg} / \mathrm{kg} \text { single } \mathrm{IV} \text { dose } \\
\mid 4.3-21.4 \\
1.6-2.7 \\
-\end{array}$ \\
\hline \multicolumn{3}{|l|}{ Human $^{\mathrm{a}}$} \\
\hline $\begin{array}{l}\text { Dose } \\
\text { CL (mL/day/kg) } \\
\text { TI/2 (day) } \\
\text { Vss (mL/kg) }\end{array}$ & $\begin{array}{l}3.6 \mathrm{mg} / \mathrm{kg} \mathrm{IV} \mathrm{q} 3 \mathrm{~W} \\
12.9 \pm 3.4 \\
3.5 \pm 0.8 \\
60 \pm 13.6\end{array}$ & $\begin{array}{l}1.8 \mathrm{mg} / \mathrm{kg} \mathrm{IV} \mathrm{q3w} \\
25.1 \\
4.43 \\
117\end{array}$ \\
\hline References & $(19,20)$ & $(21,22)$ \\
\hline
\end{tabular}

${ }^{a}$ Human PK parameters from Phase I studies in Cycle I at doses at or near MTD

\section{Linker}

An important consideration impacting the pharmacokinetics of an ADC is the stability of linker. Ideally the linker should be stable in circulation to minimize toxicity but release the active cytotoxin once it is internalized by the tumor cell. The linkers can be categorized as cleavable and non-cleavable, with varying degrees of stability (1,31). Cleavable linkers use mechanisms within the cells or cellular compartments to release active cytotoxin, such as low $\mathrm{pH}$ (acid labile linkers), glutathione levels (disulfide linkers), and lysosomal proteases (protease

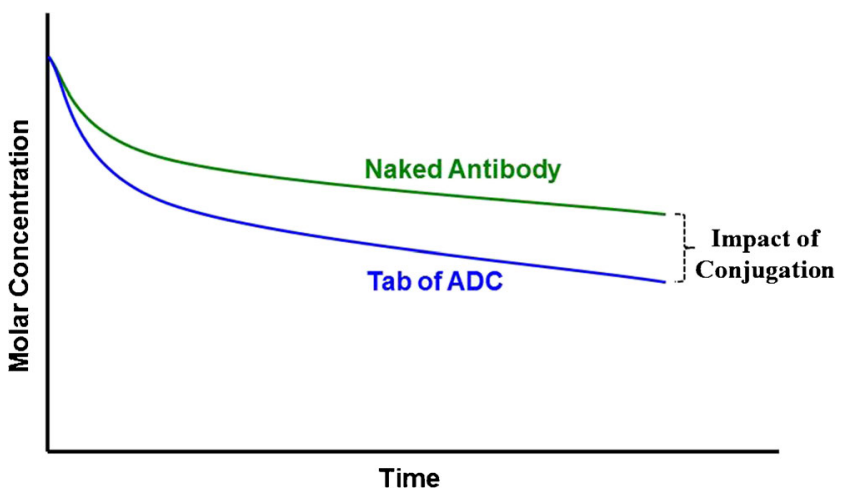

Fig. 4 Effect of conjugation on the PK profile of an antibody. cleavable linkers). In contrast, for non-cleavable linkers (e.g. thioether linkers), the entire ADC has to be degraded to release the active cytotoxin. The three advanced ADCs all use different linker types (1,31): peptide based protease cleavable linker (Adcetris ${ }^{\circledR}$, linker: MC-vc-PAB), non-cleavable thioether linker (Kadcyla ${ }^{\circledR}$, linker: $\mathrm{MCG}$ ), and acid-labile hydrazone linker (Mylotarg ${ }^{\circledR}$, linker: AcBut). The comparison of the Tab profile with the conjugated antibody profile allows for an assessment of linker stability as shown conceptually in Fig. 5, where the conjugated antibody concentrations for the more unstable linker decline much faster compared to that of the stable linker.

Different types of linkers used on the same antibody can impact its pharmacokinetics as illustrated by the following examples. The pharmacokinetics of anti-HER2 ADCs was determined in mice using disulfide linkers with different hindered structures (32). The clearance of the ADC decreased with the increase in the degree of hindrance of the disulfide linker as follows: SPDP-DM1 (least hindered) $>$ SPP-DM1 $>$ SSNPP-DM3 > SSNPP-DM4 (most hindered). The MCCDM1 linker (nonreducible thioether) had similar pharmacokinetics in this study as the SSNPP-DM4 (most hindered disulfide). Similar results were seen in other studies where the pharmacokinetics of a disulfide linker (SPP) was compared to a thioether linker (MCC) linking the cytotoxic drug DM1 to two different antibodies, anti-CD22 and anti-HER2 in rats and mice, respectively $(33,34)$. The Tab clearance for each of the antibody was similar regardless of the linker, however the ADC clearance (i.e., conjugated antibody clearance) was faster for SPP-DM1 compared to MCC-DM1, for both antiCD22 and anti-HER2 ADCs as shown in Table III. Another interesting observation was different metabolite profiles for different linker types, with the cleavable linkers releasing cytotoxic drug, and the non-cleavable linkers releasing the drug attached to an amino acid: e.g., maytansinoid ADCs with the non-cleavable linker MCC-DM1 produced only lysine-MCCDM1 whereas ADCs with cleavable linkers SPP-DM1 and SPDB-DM4 produced multiple metabolites including lysineSPP-DM1, DM1, S-methyl-DM1, S-methyl-DM1-sulfoxide and S-methyl-DM1-sulfone (for mAb-SPP-DM1), and lysine-SPDB-DM4, DM4, S-methyl-DM4, S-methyl-DM4-

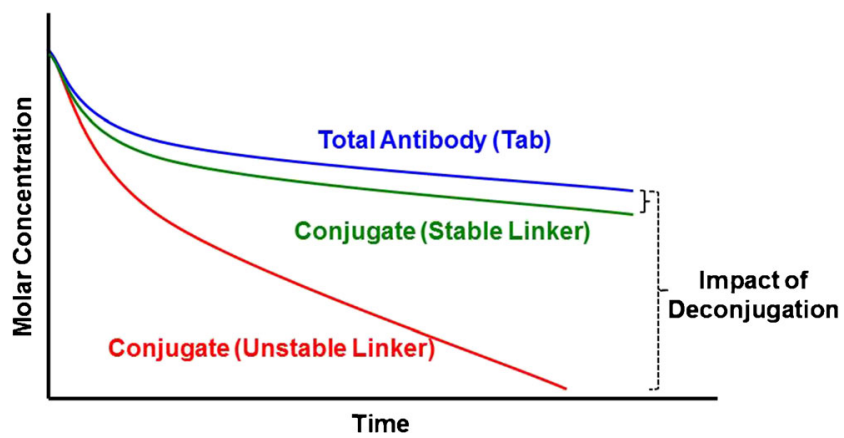

Fig. 5 PK profiles of ADCs with different linker stability. 
Table III Effect of Linker on PK of Tab and Conjugate in Mice (Anti-Her2 ADCs) and Rats (Anti-CD22 ADCs)

\begin{tabular}{|c|c|c|c|}
\hline Molecule & Linker type & Tab CL mL/day/kg & Conjugate $\mathrm{CL} \mathrm{mL/day/kg}$ \\
\hline \multicolumn{4}{|c|}{ Anti-HER2 ADC PK in mouse ${ }^{\mathrm{a}}$ : dose of $3 \mathrm{mg} / \mathrm{kg}$} \\
\hline Trastuzumab-SPP-DMI & Cleavable (disulfide) & 8.5 & 41 \\
\hline Trastuzumab-MCC-DMI (T-DMI) & Non-cleavable & 8.4 & 19 \\
\hline \multicolumn{4}{|c|}{ Anti-CD22 ADC PK in rat": dose of $2000 \mu \mathrm{g} / \mathrm{m}^{2}$ of drug (approximately $20 \mathrm{mg} / \mathrm{kg}$ of ADC) } \\
\hline CD22-SPP-DMI & Cleavable (disulfide) & 13 & 67 \\
\hline CD22-MCC-DMI & Non-cleavable & $\mid 1$ & 18 \\
\hline CD22-MC-MMAF & Non-cleavable & 11 & 29 \\
\hline CD22-MC-vc-PAB-MMAE & Cleavable (protease) & 24 & 90 \\
\hline
\end{tabular}

${ }^{\text {a }}$ References: $(33,34)$

sulfoxide and S-methyl-DM4-sulfone (for mAB-SPDB-DM4) (35). Some of these metabolites could also enhance anti-tumor activity via bystander killing mechanisms where the cytotoxic agent released in one cell diffuses to neighboring cells and exerts its effect $(34,35)$.

As highlighted in these examples, during optimization of an $\mathrm{ADC}$, the linker type needs to be carefully evaluated with regard to its stability, impact on catabolism, and how that affects its pharmacokinetics.

\section{Cytotoxic Drug}

While the cytotoxic drug does not drive the PK of the ADC per $s e$, its MOA can impact the PK drivers of efficacy and toxicity. The two main categories of cytotoxins currently being explored are microtubule inhibitors (auristatins, maytansinoids) and DNA damaging agents (calicheamicin, duocarmycins, anthracyclines, pyrrolobenzodiazepine dimers). Unlike microtubule inhibitors that preferentially kill proliferating cells, DNA damaging agents can also kill non-proliferating cells, potentially giving them a different spectrum of efficacy as well as toxicity. This could play a role in changing the PK driver of efficacy as well as toxicity and emphasizes the importance of investigating appropriate dosing regimens to optimize efficacy and minimize toxicity depending on the type of cytotoxin used. The three most advanced ADCs use different cytotoxins: calicheamicin (Mylotarg $\left.{ }^{\circledR}\right)$, auristatin (MMAE, Adcetris ${ }^{\circledR}$ ), and maytansinoid (DM1, Kadcyla $\left.{ }^{\circledR}\right)$. Most of the ADCs in the clinical pipeline also use these three types of cytotoxins: calicheamicin, DM1, DM4, MMAE (7). SG-CD33A, a novel CD33 targeting ADC with a pyrolobenzodiapine dimer and using site specific conjugation with engineered cysteines has recently entered Phase 1 trials in AML patients and it will be interesting to see the pharmacokinetic and safety profiles of this ADC with a novel cytotoxin and linker $(36,37)$. In addition to the MOA of these different cytotoxins, molecule characteristics such as permeability, metabolism, and whether it is a Pglycoprotein substrate could also play important roles in determining bystander effects and resistance development (1,31).

\section{Drug Load and Site-Specific Conjugation}

The conventional processes to conjugate linker-drugs to antibodies (lysine conjugation or conjugation to cysteines derived from reduction of inert-chain disulfide bonds) produces ADCs with heterogeneous mixtures of multiple species with different drug loads: DAR of $0-8$ (10).

Each of these DAR species could potentially have distinct PK properties and activities $(28,38)$. While in vitro potency could increase with increase in drug load per antibody, it does not always follow that in vivo efficacy and safety profiles will also improve (28). Hambelet et al. (28), made ADGs with an anti-CD30 antibody (cAC10) conjugated to MMAE with the MC-vc-PAB linker with either two, four, or eight drugs per antibody. ADCs with lower drug loads (DAR of 2 or 4) had slower clearance values, longer half-lives and were better tolerated in mice compared to an ADC with a higher drug load (DAR of 8). In addition, the DAR4 ADC showed equivalent in vivo antitumor activity to the DAR8 ADC at equal antibody doses despite having half the amount of MMAE. Similar results were seen in a rat study with trastuzumab-MCvc-PAB-MMAF conjugates (DAR of 2, 4, and 6), where the ADCS with the higher drug loads cleared faster and were less tolerated compared to conjugates with lower drug loads (39).

The next generation ADC efforts have focused on eliminating this heterogeneity by using site-specific conjugation methods to produce a more homogenous ADC to improve stability, PK, and therapeutic index, as shown in several recent studies $(17,29,40,41)$. These conjugation strategies include the use of engineered cysteines, unnatural amino acids, and enzymatic conjugation through glucotransfersases and transglutaminases (10). A recent study at Genentech using site-specific conjugation with engineered cysteines (THIOMAB ${ }^{\mathrm{TM}}$ technology) showed that chemical and structure dynamic of the conjugation site can influence the stability of the ADC (17). Three thio-trastuzumab-MC-vc-MMAE THIOMABs were generated (DAR of 1.7-1.9) using engineered cysteines at three different sites (FC-S396C, LCV205C, HC-A114G), differing in solvent accessibility and 
local charge. The conjugate at a highly solvent accessible site (Fc-S396C) was the most unstable in plasma and allowed maleimide exchange of the linker drug with reactive thiols in albumin, free cysteine, or reduced glutathione. Other ADCs using maleimide chemistry such as anti-CD30-MC-MMAF and anti-CD70-MC-MMAF have also shown adduct formation in plasma, such as albumin-MC-MMAF and cys-MCMMAF $(42,43)$. The conjugate at a partially accessible site with a positively charged environment (LC-V205C) prevented this maleimide exchange by promoting succinimide ring hydrolysis and was the most stable in plasma. The stability of the third conjugate at a partially accessible site with a neutral environment (HC-A114G) was in between the other two and showed both mechanisms. The stability of these variants corresponded with their in vivo activity with the more stable conjugate showing greater in vivo efficacy in mouse xenograft models compared to the least stable conjugate. Other site-specific conjugation methods such as use of transglutaminase have also shown that conjugation site has an impact on $\mathrm{ADC}$ stability and pharmacokinetics (44).

Conjugation can also impact tissue distribution of the antibody and several studies have shown a trend towards slightly increased hepatic uptake of ADCs $(29,30,42)$. This was seen with auristatin $(29,42)$ and calicheamicin conjugates (30). In contrast, the maytansinoids conjugated to the antibody through lysine residues (e.g. Kadcyla ${ }^{\circledR}$, SAR3419, IMGN901) showed tissue distribution profiles similar to the naked antibody $(45,46)$.

For auristatin conjugates, tissue distribution studies have been conducted using the protease cleavable MC-vc-PABMMAE linker with different drug loads (DAR 3.1 vs. DAR 1.7) and different conjugation methods (reduced interchain disulfides $v s$. site specific conjugation through engineered cysteines) (29). Since increased hepatic uptake was seen with conjugates with lower drug loads as well as different conjugation methods compared to the naked antibody, one proposed hypothesis was that higher hydrophobicity of the drug conjugates compared to the naked antibody leads to a greater clearance by the reticuloendothelial system (29). A recent study by Seattle Genetics (47) to explore increased ADC clearance with higher drug loading seen for the MC-vcauristatin linkers showed a correlation between hydrophobicity and plasma clearance. The rapid plasma clearance with higher drug loading (DAR of 8) seen with MC-vc-MMAF linker (more hydrophobic), was slightly less pronounced with MG-MMAF (slightly less hydrophobic) and was not seen with a novel auristatin T-based drug linker (AT-GLu-MDpr) which was designed to minimize hydrophobicity. In addition, the AT-Glu-MDpr linked conjugate with high drug load showed similar hepatic uptake as its parent antibody in a perfused liver system. They also showed that full reduction of the interchain disulfides of an antibody, without any drugs attached to it, did not increase clearance of the antibody. This study indicates that increased clearance and hepatic uptake could be attributed to the intrinsic hydrophobicity of the drug linker as hypothesized by several groups (29,31), and not due to destabilization of the antibody structure due to reduction of the interchain disulfides. Additional studies with different antibodies as well as in vivo tissue distribution studies are needed to confirm these interesting findings.

\section{PRECLINICAL PK STRATEGY TO EVALUATE ADCS}

At the preclinical stage, it is important to understand the pharmacokinetics of an ADC in conjunction with its in vitro and in viwo activity to gain insights into its mechanism of action and help optimize and select the right ADC. ADC PK is usually characterized in non-clinical species used for efficacy and safety studies. The choice of the species for these studies usually faces similar challenges as with the naked antibody in terms of appropriate antigen binding i.e., when some species may be a non-binding species. However, the non-antigen dependent process should be similar in binding or nonbinding species. To adequately characterize the PK of an $\mathrm{ADC}$, it is critical to have the appropriate tools. As discussed previously, there have been great advances in the types of analytical methods to measure the different components of the ADCs like the total antibody, conjugated and unconjugated drug, DAR distribution, catabolites and metabolites in various matrices such as plasma, bile, and tissues from in vitro or in viwo studies depending on the different stages of drug development (11-13). Additional work is still needed to better understand the best analytes to use for exposure-response correlations and to better understand in vitro-in vivo correlations as well as crossspecies correlations.

Types of studies that can be done preclinically to characterize the PK of ADCs include the following:

i. In vitro stability studies in plasma from different species to understand linker stability as well as mechanisms of deconjugation across species.

ii. In vitro catabolism studies to determine the types of catabolites/metabolites formed and whether they have any activity in in-vitro cell potency assays.

iii. In viwo $\mathrm{PK}$ and exposure of the various analytes in the efficacy and toxicity species to characterize the PK, determine PK drivers of efficacy/toxicity, establish in vitro-in vivo correlations of stability and mechanisms of deconjugation/catabolism.

iv. Biodistribution studies to look for tumor and normal tissue uptake (specific or non-specific), and in viwo catabolite profiles in various tissues, including understanding any contribution of catabolites to any bystander effects. 
v. In vitro potency, CYP, and transporter profiling of the cytotoxic drug to evaluate the risk of possible drug-drug interactions in the clinic.

vi. Utilize in vivo exposure data at the efficacious and toxic doses to estimate therapeutic index.

vii. Prediction of human PK to estimate efficacious dose and schedule in patients.

In addition to studies to characterize the PK of ADCs, it is also important to integrate PK, efficacy and toxicity data to answer the key question on exposure-response relationships and translation from preclinical species to patients. Model based approaches can be used to integrate this data and further our mechanistic understanding of the pharmacology. Depending on the stage of development, different questions become important (e.g., questions on target selection, antibody affinity, linker stability, E-R relationship) and the type of model employed will depend on the type of question that needs to be addressed. For example: early on in development, systems pharmacology models can be used to enable target selection, whereas classic PKPD models can be used later on to understand exposure-response relationships as well as translational PKPD to predict human PK as well as efficacious dose and dose regimens in patients. The modeling of ADC PK is complicated due to the its multiple elimination pathways including deconjugation and catabolism, as well as multiple analytes with their distinct PK properties (conjugated antibody with multiple DAR species, unconjugated drug, unconjugated antibody, etc.). Tremendous efforts are ongoing in developing PK/PD models to guide the development of ADGs. Some of the models that have been proposed include semi-mechanistic PK models using a series of transit compartments to describe the deconjugation process from higher to lower DAR species $(48,49)$, simplified models with a one-step deconjugation process $(48,50)$, target-mediated drug disposition models (51), and multi-scale mechanistic PK/PD models $(52,53)$. A key limitation for the use of some of these models is the availability of appropriate data, or tools to obtain that information. However, as more clinical data becomes available and tools to obtain these types of data improve, sophisticated models can be developed and applied to expand our understanding of the key analytes that correlate with efficacy and safety.

\section{CONCLUSION}

The ADC field is rapidly expanding with many molecules at various stages of development. With limited clinic data on a small number of ADCs, the guidelines and strategies for developing these molecules are still evolving. This review has highlighted several areas where PK of an ADC makes an impact on its activity. There have been huge strides in this field, especially with new linker technology, site-specific conjugation techniques to create more homogenous ADCs, as well as tremendous advances in novel bionalytical tools to measure PK of various analytes and methods to integrate the available PKPD information. Nevertheless, many questions still remain on PK drivers of efficacy and toxicity, optimal design features of an ADC, translation of PKPD from non-clinical species to patients, and optimal doses and dosing regimens in the clinic. As more clinical data becomes available and our understanding of the PK of ADCs with different types of toxins, linkers and antibody formats improves, we can tackle some of these outstanding questions.

\section{ACKNOWLEDGEMENTS AND DISCLOSURES}

All authors are employees of Genentech, a member of the Roche Group, and hold financial interest in Hoffman-La Roche.

Open Access This article is distributed under the terms of the Creative Commons Attribution License which permits any use, distribution, and reproduction in any medium, provided the original author(s) and the source are credited.

\section{REFERENCES}

1. Sievers EL, Senter PD. Antibody-drug conjugates in cancer therapy. Annu Rev Med. 2013;64:15-29.

2. Lambert JM. Drug-conjugated antibodies for the treatment of cancer. Br J Clin Pharmacol. 2012;76(2):248-62.

3. Sliwkowski MX, Mellman I. Antibody therapeutics in cancer. Science. 2013;341:1192-8.

4. Carter PJ, Senter PD. Antibody-drug conjugates for cancer Therapy. Cancer J. 2008; 154-169.

5. Prabhu S, Boswell CA, Leipold D, Khawli LA, Li D, Lu D, et al. Ther Deliv. 201 1;2(6):769-91.

6. Alley SC, Okeley NM, Senter PD. Antibody-drug conjugates: targeted drug delivery for cancer. Curr Opin Chem Biol. 2010;14: 529-37.

7. Mullard A. Maturing antibody-drug conjugate pipeline hits 30. Nat Rev Drug Discov. 2013;12:329-32.

8. Beck A, Reichert JM. Antibody-drug conjugates: present and future. mAbs. 2014;15-17.

9. Lin K, Tibbitts J. Pharmacokinetic considerations for antibody drug conjugates. Pharm Res. 2012;29:2354-66.

10. Panowski S, Bhakta S, Raab H, Polakis P, Junutula JR. Site-specific antibody drug conjugates for cancer therapy. mAbs. 2014;6(1):34 45.

11. Kaur S, Xu K, Saad OM, Dere FG, Carrasco-Triguero M. Bioanalytical assay strategies for the development of antibody-drug conjugate biotherapeutics. Bioanalysis. 2013;5(2):201-26.

12. Alley SC, Anderson KE. Analytical and bioanalytical technologies for characterizing antibody-drug conjugates. Curr Opin Chem Biol. 2013;17:406-11.

13. Gorovits B, Alley SC, Bilic S, Booth B, Kaur S, Oldfield P, et al. Bioanalysis of antibody-drug conjugates: American association of pharmaceutical scientists antibody-drug conjugate working group position paper. Bioanalysis. 2013;5(9):997-1006. 
14. Xu K, Liu L, Saad OM, Baudys J, Williams L, Leipold D, et al. Characterization of intact antibody-drug conjugates from plasma/ serum in vivo by affinity capture capillary liquid chromatographymass spectrometry. Anal Biochem. 2011;412:56-66.

15. Xu K, Liu L, Dere R, Mai E, Erickson R, Hendricks A, et al. Characterization of the drug-to-antibody ratio distribution for antibody-drug conjugates in plasma/serum. Bioanalysis. 2013;5(9): $1057-71$.

16. Hengel SM, Sanderson R, Valliere-Douglass J, Nicholas N, Leiske C, Alley SC. Measurement of in vivo drug load distribution of cysteinelinked antibody-drug conjugates using microscale liquid chromatography mass spectrometry. Anal Chem. 2014;86(7):3420-5.

17. Shen BQ $\mathrm{Xu} \mathrm{K}$, Liu L, Raab H, Bhakta S, Kenrick M, et al. Conjugation site modulates the in vivo stability and therapeutic activity of antibody-drug conjugates. Nat Biotechnol. 2012;30:1849.

18. Erickson HK, Park PU, Widdison WC, Kovtun YV, Garrett LM, Hoffman K, et al. Antibody-maytansinoid conjugates are activated in targeted cancer cells by lysosomal degradation and linker-dependent intracellular processing. Cancer Res. 2006;66(8):4426-33.

19. Poon KA, Flagella K, Beyer J, Tibbitts J, Kaur S, Saad O, et al. Preclinical safety profile of trastuzumab emtansine (T-DMl): mechanism of action of its cytotoxic component retained with improved tolerability. Toxicol Appl Pharmacol. 2013;273(2):298-313.

20. Krop IE, Beeram M, Modi S, Jones SF, Holden SN, Yu W, et al. Phase I study of trastuzumab-DM1, an HER2 antibody-drug conjugate, given every 3 weeks to patients with HER2-positive metastatic breast cancer. J Clin Oncol. 2010;28(16):2698-704.

21. Adcetris. EMA CHMP Assessment report. 2012.

22. Younes A, Bartlett NL, Leonard JP, Kennedy DA, Lynch CM, Sievers EL, et al. Brentuximab vedotin (SGN-35) for relapsed CD30-positive lymphomas. N Engl J Med. 2010;363(19):1812-21.

23. Wang W, Wang EQ, Balthasar JP. Monoclonal antibody pharmacokinetics and pharmacodynamics. Clin Pharmacol Ther. 2008;84(5):548-58.

24. Deng R, Jin F, Prabhu S, Iyer S. Monoclonal antibodies: what are the pharmacokinetic and pharmacodynamics considerations for drug development? Expert Opin Drug Metab Toxicol. 2012;8(2):141-60.

25. Glassman PM, Balthasar JP. Mechanistic considerations for the use of monoclonal antibodies for cancer therapy. Cancer Biol Med. 2014;11(1):20-33.

26. Pastuskovas CV, Mallet W, Clark S, Kenrick M, Majidy M, Schweiger M, et al. Effect of immune complex formation on the distribution of a novel antibody to the ovarian tumor antigen CA125. Drug Metab Dispos. 2010;38:2309-19.

27. Lovdal T, Anderson E, Brech A, Berg T. Fc receptor mediated endocytosis of small soluble immunoglobulin $\mathrm{G}$ immune complexes in Kupffer and endothelial cells from rat liver. J Cell Sci. 2000;113: 3255-66.

28. Hamblett KJ, Senter PD, Chace DF, Sun MM, Lenox J, Cerveny $\mathrm{CG}$, et al. Effects of drug loading on the antitumor activity of a monoclonal antibody drug conjugate. Clin Cancer Res. 2004;10: 7063-70.

29. Boswell CA, Mundo EE, Zhang C, Bumbaca D, Valle NR, Kozak $\mathrm{KR}$, et al. Impact of drug conjugation on pharmacokinetics and tissue distribution of anti-STEAP1 antibody-drug conjugates in rats. Bioconjug Chem. 2011;22(10):1994-2004.

30. Herbertson RA, Tebbutt NG, Lee FT, MacFarlane DJ, Chappell B, Micallef N, et al. Phase I biodistribution and pharmacokinetic study of Lewis Y-targeting immunoconjugate CMD-193 in patients with advanced epithelial cancers. Clin Cancer Res. 2009;15(21):6709-15.

31. Flygare JA, Pillow TH, Aristoff P. Antibody-drug conjugates for the treatment of cancer. Chem Biol Drug Des. 2013;81:113-21.

32. Lewis Phillips GD, Li G, Dugger DL, Crocker LM, Parsons KL, Mai E, et al. Targeting HER2-positive breast cancer with trastuzumab-
DM1, an antibody-cytotoxic drug conjugate. Cancer Res. 2008;68(22):9280-90.

33. Polson AG, Calemine-Fenaux J, Chan P, Chang W, Christensen E, Clark S, et al. Antibody-drug conjugates for the treatment of nonHodgkin's lymphoma: target and linker-drug selection. Cancer Res. 2009;69(6):2358-64.

34. Erickson HK, Lewis Phillips GD, Leipold DD, Provenzano CA, Mai E, Johnson HA, et al. The effect of different linkers on target cell catabolism and pharmacokinetics/pharmacodynamics of trastuzumab maytansinoid conjugates. Mol Cancer Ther. 2012;11(5):1133-42.

35. Sun X, Widdison W, Mayo M, Wilhelm S, Leece B, Chari R, et al. Design of antibody-maytansinoid conjugates allows for efficient detoxification via liver metabolism. Bioconjug Chem. 2011;22(4):728-35.

36. Kung Sutherland MS, Walter RB, Jeffrey SC, Burke PJ, Yu C, Kostner H, et al. SGN-CD33A: a novel CD33-targeting antibodydrug conjugate using a pyrrolobenzodiazepine dimer is active in models of drug-resistant AML. Blood. 2013;122(8):1455-63.

37. A Safety Study of SGN-CD33A in AML Patients. ClinicalTrials.gov.

38. Okeley NM, Alley SC, Senter PD. Advancing antibody drug conjugation from the laboratory to a clinically approved anticancer drug. Hematol Oncol Clin N Am. 2014;28:13-25.

39. Leipold DD, Jumbe N, Duggar D, Crocker L, Leach W, Sliwkowski MX, et al. Trastuzumab-Mc-vc-PAB-MMAF: the effects of the drug: antibody ratio (DAR) on efficacy, toxicity and pharmacokinetics. AACR Annual Meeting 2007, Abstract \#1551.

40. Junutula JR, Raab H, Clark S, Bhakta S, Leipold DD, Weir $\mathrm{S}$, et al. Site-specific conjugation of a cytotoxic drug to an antibody improves the therapeutic index. Nat Biotechnol. 2008;26:925-32.

41. Junutula JR, Flagella KM, Graham RA, Parsons KL, Ha E, Raab $\mathrm{H}$, et al. Engineered thio-trastuzumab-DMl conjugate with an improved therapeutic index to target human epidermal growth factor receptor 2-positive breast cancer. Clin Cancer Res. 2010;16:4769-78.

42. Alley SC, Zhang X, Okeley NM, Anderson M, Law CL, Senter PD, et al. The pharmacologic basis for antibody-auristatin conjugate activity. J Pharmacol Exp Ther. 2009;330(3):932-8.

43. Alley SC, Benjamin DR, Jeffrey SC, Okeley NM, Meyer DL, Sanderson RJ, et al. Contribution of linker stability to the activities of anticancer immunoconjugates. Bioconjug Chem. 2008;19(3):759-65.

44. Strop P, Liu SH, Dorywalska M, Delaria K, Dushin RG, Tran TT, et al. Location matters: site of conjugation modulates stability and pharmacokinetics of antibody drug conjugates. Chem Biol. 2013;20(2):161-7.

45. Erickson HK, Lambert JM. ADME of antibody-maytansinoid conjugates. AAPS J. 2012;14(4):799-805.

46. Shen BQ Bumbaca D, Saad O, Yue Q Pastuskovas GV, Khojasteh SC, et al. Catabolic fate and pharmacokinetic characterization of trastuzumab emtansine (T-DM1): an emphasis on preclinical and clinical catabolism. Curr Drug Metab. 2012;13(7):901-10.

47. Doronina SO, Setter JR, Hunter JH, Bovee TD, Anderson ME, Jonas M, et al. Elucidating the role of drug-linker hydrophobicity in the disposition of antibody-drug conjugates. AACR Annual Meeting 2014, Abstract \#4470.

48. Bender B, Leipold DD, Xu K, Shen BQ, Tibbitts J, Friberg LE. A mechanistic pharmacokinetic model elucidating the disposition of trastuzumab emtansine (T-DM1), an Antibody-Drug Conjugate (ADG) for Treatment of Metastatic Breast Cancer. AAPS J. 2014; Epub ahead of print.

49. Chudasama VL, Schaedeli Stark F, Harrold JM, Tibbitts J, Girish SR, Gupta M, et al. Semi-mechanistic population pharmacokinetic model of multivalent trastuzumab emtansine in patients with metastatic breast cancer. Clin Pharmacol Ther. 2012;92(4):520-7. 
50. Lu D, Girish S, Gao Y, Wang B, Yi JH, Guardino E, et al. Population pharmacokinetics of trastuzumab emtansine (T-DMl), a HER2-targeted antibody-drug conjugate, in patients with HER2positive metastatic breast cancer: clinical implications of the effect of covariates. Cancer Chemother Pharmacol. 2014; Epub ahead of print.

51. Gibiansky L, Gibiansky E. Target-mediated drug disposition model and its approximations for antibody-drug conjugates. J Pharmacokinet Pharmacodyn. 2014;41(1):35-47.
52. Shah DK, Haddish-Berhane N, Betts A. Bench to bedside translation of antibody drug conjugates using a multiscale mechanistic PK/PD model: a case study with brentuximab-vedotin. J Pharmacokinet Pharmacodyn. 2012;39(6):643-59.

53. Haddish-Berhane N, Shah DK, Ma D, Leal M, Gerber HP, Sapra $\mathrm{P}$, et al. On translation of antibody drug conjugates efficacy from mouse experimental tumors to the clinic: a PK/ PD approach. J Pharmacokinet Pharmacodyn. 2013;40(5):55771. 